\title{
A NEW MEASL'REMENT OF THE GEOMETRY OF SPACE
}

\author{
Edwin D. Loh \\ Physics Department \\ Princeton University \\ P. O. Box 708 \\ Princeton, New Jersey 08544 \\ USA
}

\begin{abstract}
This paper discusses the recent measurement of the number of galaxies vs. redshift and flux and presents new results pertaining to the two dimensionless geometrical quantities that describe the geometry of the conventional big-bang cosmology, the density parameter $\Omega$ and the dimensionless form $\lambda=\Lambda /\left(3 \mathrm{H}_{0}{ }^{2}\right)$ of the cosmological constant. In contrast to the classical redshift-magnitude test as applied to the brightest galaxies in clusters, this new method is able to separate the effects of evolution from geometrical effects and is therefore able to measure the geometry of space. The $95 \%$ confidence limits are $\Omega-\lambda=0.9_{-05}^{+07}$ and $-1.5<\Omega+\lambda<7.1$. The principal conclusions are these: (1) For both $\lambda=0$ and inflationary models of the universe, this measurement and primordial nucleosynthesis imply a large density of nonbaryonic matter. (2) Hubble's constant $H_{0}$ and the age of the universe $\tau$ are constrained by $0.60<H_{0} \tau<0.88$ (95\% confidence).
\end{abstract}

\section{INTRODUCTION}

Measuring the geometry of space has been hindered because comparing quantities over large distances $\sim \mathrm{H}_{0}^{-1}$ to measure the geometry entails comparing them over long times $\sim H_{0}^{-1}$ and uncertain histories. Recently Loh and Spillar (1986b) measured the joint distribution of redshift and flux for a flux-limited sample of galaxies. They argue that the geometrical effects and the effects of luminosity evolution are separable and the evolution of the number of galaxies is small, and therefore they have measured the volume element as a function of redshift, a geometrical quantity. Here I review these results briefly and present the current constraints on the geometry of space.

\section{NUMBER OF GALAXIES vs. REDSHIFT AND FLUX}

One measures the flux and redshift $z$ of every galaxy with a greater than minimum flux in a pencil beam of solid angle $d \omega$. The number of galaxies with a given redshift and flux is the product of the volume and the density.

$$
\text { Volume }=H_{0}^{-3} a^{3} \frac{r^{2} d r}{\sqrt{ }\left(1-K r^{2}\right)} d \omega=H_{0}^{-3} a^{3} A(z ; \Omega, \lambda) z^{2} d z d \omega
$$


$a=(1+z)^{-1}$, and $r$ is the comoving coordinate. The density in space and luminosity $L$ is the Schechter function

$$
\phi(L) d L=a^{-3} \phi^{*} e^{-x} x^{\alpha} d x, \quad x=L / L^{*}, \quad \text { and } \alpha \approx-1.25
$$

From the measured number of galaxies one extracts the quantity $A \phi^{*} H_{0}{ }^{-3}$. The quantity $A(z ; \Omega, \lambda)$, the volume element relative to $a^{3} H_{0}{ }^{-3} z^{2} d z d \omega$, contains the geometrical information. The volume element and hence the observed number of galaxies is a strong function of $\Omega$; eg., $A(0.75 ; 0,0) / A(0.75 ; 1,0)=1.9$.

The technical advance that enables this measurement is the photometric method (Loh and Spillar 1986a) for measuring redshifts, which Baum invented 30 years ago. In approximate terms one determines the redshift of every galaxy in a field by finding the wavelength of the $400 \mathrm{~nm}$ break with six broad-band filters. In 3 hours on the Wyoming $2.3 \mathrm{~m}$ telescope, we obtain the redshifts of 200 galaxies, for which the median redshift is 0.5. Spectroscopy yields redshifts with higher precision, but photometry is faster.

It is instructive to compare this measurement of geometry with the classical redshiftmagnitude test of the brightest galaxy in rich clusters (Sandage 1961). For the redshiftmagnitude test, a $10 \%$ difference in $\Omega$ implies a $3 \%$ difference in the luminosity at $z=$ 0.75 . Stellar evolution can shift the apparent value of $\Omega$ by $\Delta \Omega \geq 2.8$ (Tinsley and Gunn 1975). Mergers of galaxies can shift $\Omega$ by $\Delta \Omega \approx-2.2$ (Ostriker and Tremaine 1975). Therefore the systematic corrections are large and uncertain.

For this the redshift-volume test, a $10 \%$ difference in $\Omega$ implies a $5.5 \%$ difference in the number of galaxies. Loh and Spillar (1986b) measured the correction for luminosity evolution; $\Delta \Omega<0.2$ (95\% confidence). The correction for mergers is $\Delta \Omega \approx 0.05$ from Toomre's (1977) estimates of merging NGC galaxies.

With the present amount of data, the systematic errors are smaller than the random errors. The largest systematic error in $\phi^{*} A$ at $z=0.75$, due to possible spectral evolution, is less than $11 \%(2 \sigma)$. For comparison, the random errors are $21 \%$. See Loh and Spillar (1986b) for a more complete discussion.

Several comments on the redshift-volume test are important:

1. Numbers of galaxies are dimensionless as are $\Omega$ and $\lambda$. To make this measurement one need not transfer distant times and lengths to laboratory standards as is the case for measuring dimensional quantities such as Hubble's constant.

2. For the interpretation of this measurement to be secure, the number of galaxies in a comoving box must be conserved. That this assumption is close to the truth can be seen from two observations. (a) It is difficult to prevent large galaxies from forming since the formation time is approximately the dynamical time, which is $0.2 \mathrm{Gyr}$ for the Galaxy.

(b) Galaxies live a long time because stars do.

3. 'First-order' evolution, where all galaxies brighten by the same factor, does not affect the measurement of $\phi^{*} A$.

4. Even though galaxies are observed, they are used solely as benchmarks of space. Therefore this experiment is sensitive to all kinds of matter, be it luminous or dark.

\section{RESULTS}

Fitting 1000 galaxies in a solid angle $24 \mu$ sr from Loh and Spillar (1986b), one finds these limits for the independent linear combinations of $\Omega$ and $\lambda$

$$
\Omega-\lambda=0.9_{-0.5}^{+0.7} \text { and }-1.5<\Omega+\lambda<7.1
$$




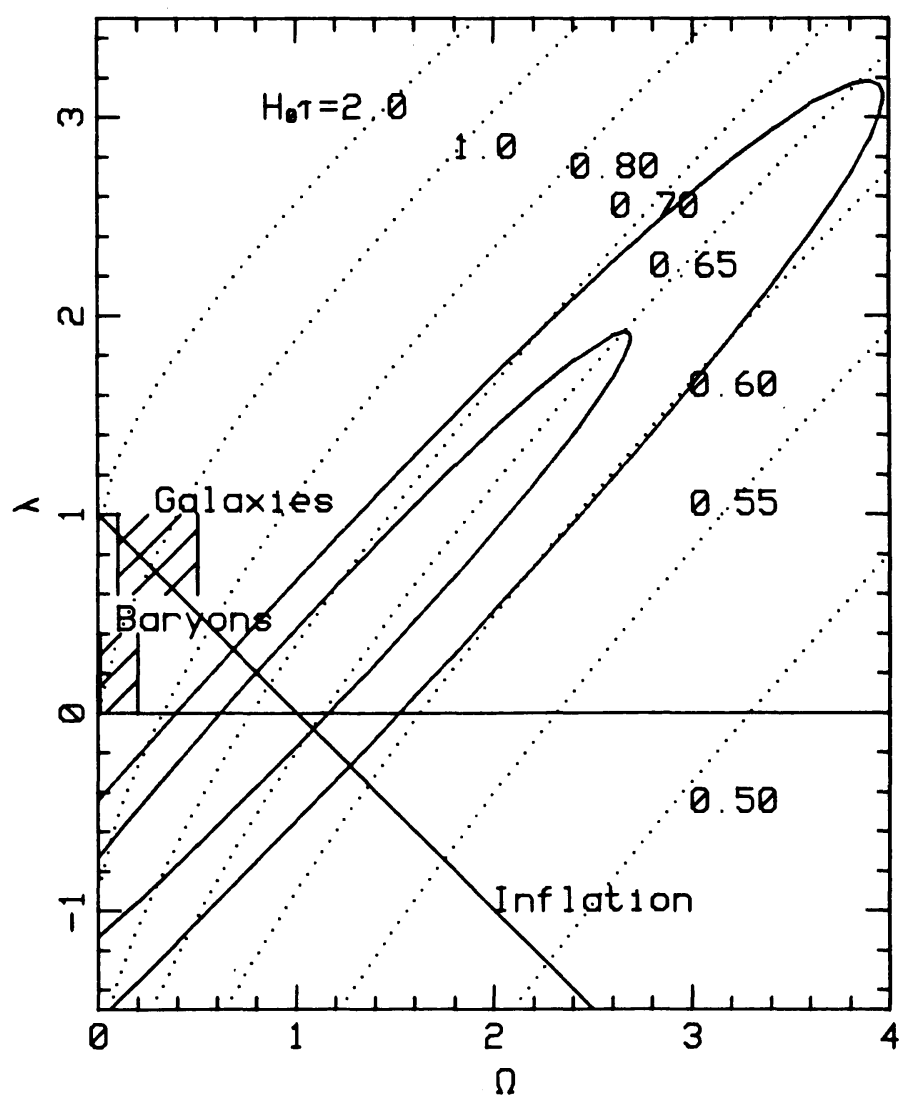

Fig 1.- The $95 \%$ and $67 \%$ confidence regions in the $\Omega, \lambda$ plane. Other measurements (at the $95 \%$ confidence level) of the mass density are shown as hatched regions: Yang et al. (1984) find that $0.01<\Omega_{\text {baryon }}<0.2$. Peebles (1984) finds that the mass associated with galaxies is bound by $0.1<$ $\Omega_{\mathrm{gal}}<0.5$. The prediction of the inflationary theory is shown as the diagonal line. Loci (dotted lines) of models with equal $H_{0} \tau$ are shown.

for a homogeneous and isotropic universe with pressureless matter. $95 \%$ confidence limits are used here and in the following. See Fig. 1.

\subsection{Mass Density}

Nonbaryonic matter exists in abundance if $\lambda=0$ or space is flat $(K=\Omega+\lambda-1=0)$. From the abundances of the light elements and the theory of nucleosynthesis, Yang et al. (1984) conclude that $0.01<\Omega_{\text {baryon }}<0.2$. This experiment implies that $\Omega=0.9_{-0.5}^{+0.7}$ if $\lambda=0$ and $\Omega=0.9_{-0.2}^{+0.4}$ if $K=0$.

Dynamical measurements of galaxies, which are sensitive only to the matter associated with galaxies, yield $0.1<\Omega_{\mathrm{gal}}<0.5$ (Peebles 1984). If $K=0$, then galaxies do not trace the mass. 


\subsection{Hubble's Constant $H_{0}$ and the Age of the Universe $\tau$}

This measurement implies

$$
0.60<H_{0} \tau<0.88, \text { or } 6.0 \mathrm{Gyr}<h \tau<8.8 \mathrm{Gyr} \text {, }
$$

$H_{0}=h 100 \mathrm{~km} / \mathrm{sec} / \mathrm{Mpc}$. The $95 \%$ confidence limits span a range of $47 \%$. The age as measured by nuclear clocks is accurate to a factor of 2 (Fowler and Meisl 1985). For the age of globular clusters the $95 \%$ confidence limits, which account for measurement errors only, span a factor of 2 , and systematic corrections amounting to $40 \%$ are possible (Iben and Renzini 1984). There is a well known disagreement in the value of Hubble's constant of a factor of 2. Therefore this measurement of $H_{0} \tau$ is more accurate and precise than the separate measurements of $H_{0}$ and $\tau$.

\subsection{Cosmology}

At the present this measurement is not precise enough to choose between $\Omega=1$ and $\Omega=0$ models of the universe without assumptions about the cosmological constant. However if one accepts the prediction $K=0$ of the inflationary theory because it provides an unforced explanation for the isotropy of the cosmic background radiation, then this experiment yields $\Omega=0.9_{-0.2}^{+0.4}$ and $\lambda=-0.1_{-0.4}^{+0.2}$. This experiment with the theory of inflation is consistent with the Einstein-deSitter model $(\Omega=1$ and $\lambda=0)$, which requires no fine tuning at large $z$.

\section{REFERENCES}

Fowler, W. A. and Meisl, C. C. 1985, Caltech preprint.

Iben, I. and Renzini, A. 1984, Phys. Rep., 105, 329.

Loh, E. D. and Spillar, E. J. 1986a, Ap. J., 303, 154.

Loh, E. D. and Spillar, E. J. 1986b, Ap. J. Lett., 307, L1.

Ostriker, J. P. and Tremaine 1975, Ap. J. Lett., 202 , L113.

Peebles, P. J. E. 1984, Ap. J., 284, 439.

Sandage, A. 1961, Ap. J., 133, 355.

Tinsley, B. M. and Gunn, J. E. 1975, Ap. J., 203, 52.

Toomre, A. 1977, in Evolution of Galaxies and Stellar Populations, ed. B. M. Tinsley and

R. Larson (New Haven: Yale University Observatory), p. 401.

Yang, J., Turner, M. S., Steigman, G., Schramm, D. N., and Olive, K. A. 1984, Ap. J., 281, 493. 


\section{DISCUSSION}

ELLIS: The validity of this method rests ultimately on the accuracy of photometric redshifts. Spectroscopy shows most faint galaxies have weak or non-existent $4000 \dot{A}$ breaks. Thus I imagine significant errors could arise in their estimation from multi-colour methods. Can you check the values, or even the redshift distributions with those obtained spectroscopically?

LOH: We have compared spectroscopic and photometric redshifts for the cluster 0024+1654 (Ap. J. 303, 154, 1986), which contains about 20 blue galaxies. It is true that the errors in the photometric redshifts are larger for the blue galaxies. However, we have shown that there is no bias in the photometric redshifts, and that is all that is needed for the measurement of the volume. We also show that the errors in the photometric redshifts are estimated accurately. Furthermore, the photometric redshifts are sufficiently accurate that any systematic errors that they introduce will be small compared to the random errors (Ap. J. (Letters) 307, L1, 1986). 